\title{
The prevalence of non organ specific and thyroid autoimmunity in patients with polycystic ovary syndrome
}

\author{
Jana Petrikovaa ${ }^{a}$, Ivica Lazurovaa ${ }^{a}$ Ingrid Draveckaa , Jana Vrbikova ${ }^{b}$, Darina Kozakovac, Jana Figurovaa , Zuzana Vaczy ${ }^{d}$, \\ Jan Rosochad
}

Background. Polycystic ovary syndrome (PCOS) is commonly associated with endocrine, metabolic, cardiovascular and other morbidities. However its association with autoimmune diseases is still controversial.

Aim. The aim of this study was to assess the prevalence of non organ-specific and antithyroid, antibodies in PCOS women compared to healthy controls.

Methods. The study included 152 women with PCOS and 76 healthy controls for the evaluation of non organ-specific autoimmunity and 64 PCOS and 68 controls for the study of organ-specific autoimmunity. All sera were tested for autoantibodies.using the ELISA method.

Results. There were no significant differences in the prevalence of ANA, SSA, SSB, anti-dsDNA, anti-RNP, ANCA/MPO or ANCA/PR3 between PCOS and controls. The prevalence of ACLA IgG was higher in controls than PCOS (5.4\% v.s. $0 \%$, $P=0.011)$. Patients had a higher prevalence of anti-TPO antibodies $(18.75 \%$ v.s. $7.35 \%, P=0.045)$ and slightly but not significantly higher prevalence of autoimmune thyroiditis (18.75\% v.s. $10.29 \%)$ than controls.

Conclusion. The prevalence of non organ-specific autoantibodies in PCOS women is low and similar to controls. On the other hand, we found a slightly higher prevalence of thyroid autoimmunity in PCOS women.

Key words: autoimmunity, autoimmune thyroid disease, polycystic ovary syndrome, systemic autoantibodies, hyperandrogenism

Received: August 5, 2014; Accepted: November 13, 2014; Available online: December 5, 2014

http://dx.doi.org/10.5507/bp.2014.062

${ }^{a}{ }^{\text {st }}$ Department of Internal Medicine, Medical Faculty, P. J. Safarik University Kosice, Slovak Republic

bInstitute of Endocrinology, Prague, Czech Republic

'National Institute of Rheumatic Disesases, Piestany, Slovak Republic

${ }^{d}$ Associated Tissue Bank, Medical Faculty, P. J. Safarik University Kosice, Slovak Republic

Corresponding author: Ivica Lazurova, Email: ivica.lazurova@upjs.sk

\section{INTRODUCTION}

Polycystic ovary syndrome (PCOS) is the most common endocrine disorder in women of child-bearing age. It is typically identified during the early reproductive years or adolescence and its prevalence is as high as $15 \%$ when the broader Rotterdam criteria are applied ${ }^{1}$ and about $10 \%$ according to the criteria of the Androgen Excess Society (AES) $\left(\right.$ ref. $\left.^{2}\right)$. PCOS is associated with significant endocrine, metabolic, cardiovascular, reproductive as well as psychiatric morbidities ${ }^{3,4}$.

The etiology of PCOS remains obscure but evidence exists for a multifactorial origin with a genetic predisposition. An autoimmune mechanism has been suggested in some cases of PCOS especially those with other autoimmune disorders ${ }^{5,6}$. Unfortunately the heterogeneity of the syndrome, variety of ovarian antigens as well as antibody tests have led to conflicting results.

It is well accepted that sex hormones (estrogens and androgens) may play a significant role in the mosaic of autoimmunity ${ }^{7}$. High levels of androgens are presented in patients who suffer from PCOS seeming to have a protec- tive role against the development of autoimmune diseases; however several mechanisms related to estrogen effects on the immune system oppose this activity. Estrogens increase the secretion of IL-4 in Th2 lymphocytes, IL-1 in monocytes, IL-6 in T-lymphocytes and interferon- $\gamma$ in Th1 cells and their stimulatory effect on the immune system was inhibited by progesterone $\left(\right.$ ref. $\left.^{8-10}\right)$. Patients with PCOS present low levels of progesterone due to oligo/ anovulation leading to over-stimulation of the immune system and to autoantibody production. Whether the estrogen or androgen component more strongly influence the immune system, is still unknown. Therefore, we suggest, this unique hormonal equilibrium in women with PCOS could be a suitable model for studying the hormonal influence on autoimmune diseases.

In the first part of this study we examined the prevalence of non-organ specific autoimmunity in PCOS women and compared it with non PCOS controls. Secondly we studied the occurrence of thyroid autoimmunity and autoimmune thyroid disease. 


\section{SUBJECTS AND METHODS}

The study included 152 women with PCOS that were primarily identified in an outpatient department of gynecology, endocrinology and diabetology and subsequently examined in the $1^{\text {st }}$ internal medicine department, University Hospital of Louis Pasteur, Kosice, Slovak Republic. The average age was $30.23 \pm 6.7$ (range 14-50 years). All patients fulfilled the Rotterdam diagnostic criteria for PCOS ${ }^{1}$. The exclusion criteria were history of known autoimmune disease and concurrent thyroid dysfunction. Other pathologic conditions which might influence menstrual cycle including pituitary diseases (hyperprolactionemia, hypopituitarism etc.), adrenal disorders and severe comorbidities were ruled out by careful examination. Among all PCOS women $49 \%$ of them had clinical hyperandrogenism manifested as hirsutism, 51\% of patients had no hirsutism. $26 \%$ had normal regular menstrual cycle and $74 \%$ presented with menstrual irregularities such as oligomenorrhea or amenorrhea. According to body weight $42 \%$ of patients had normal BMI, $19 \%$ were overweight, and $39 \%$ were obese.

The control group consisted of 76 healthy regularly menstruating randomly selected age matched women with no history of autoimmune disease or apparent hyperandrogenism. The average age was $29 \pm 4$ (range 22-45 years). None of them was taking hormonal contraception. All subjects signed an informed consent. All procedures followed were in accordance with the ethical standards of the responsible committee on human experimentation (institutional and national) and with the Helsinki Declaration of 1975, as revised in 2008.

The study was divided into 2 parts: 1 . organ non-specific autoimmunity in PCOS patients and 2. Organ specific, i.e. anti-thyroidal autoimmunity in PCOS patients.

\section{Laboratory assessments}

Blood samples were taken in an early follicular phase of a menstrual cycle. In case of amenorrhea, blood was taken after excluding pregnancy. Hormone levels of sex hormone binding globulin (SHBG), luteinizing hormone $(\mathrm{LH})$, and follicule-stimulating hormone (FSH) were determined using chemiluminiscent immunoassay (analyzer Architect, module C). Free testosterone (fTST) was detected by radio-imunoassay using commercially available kits of DiaSource.

\section{Study of non organ-specific autoimmunity}

In this part of the study, blood samples were collected to assess a wide spectrum of non organ specific autoantibodies, such as: antinuclear (ANA), extractable nuclear (ENA), double strained DNA (dsDNA) antibodies, antibodies against ribonucleoprotein (RNP) and vasculitis associated antibodies, such as antineutrophil cytoplasmic (ANCA) and anticardiolipin antibodies (ACLA).

Serum was obtained by centrifugation on centrifuge Capricorn, in $3 \mathrm{~g}$ during $7 \mathrm{~min}$ and consequently frozen in minus $40^{\circ} \mathrm{C}$. In parallel, a large profile belonging to 2 groups of autoantibodies was tested in all sera from the first study.
ANA/Hep2 IFT screening: screening for autoantibodies implemented in all sera using an indirect immunofluorescence test (IFT) (diagnostic kit Hep-2 cell IFA Kit, serum dilution 1:80). Only sera positive in ANA/Hep2 IFT were further evaluated by ELISA on 8S ELISA ANA screening (ANA ELISA diagnostic kit 8S, dilution of sera 1:101, a positive reaction $>$ cut off).

dsDNA screen performed by ELISA method (diagnostic kit AIDA dsDNA-G, Germany, dilution of sera 1:101, positive titer $>18 \mathrm{U} / \mathrm{mL}$ )

ANCA screen: performed by ELISA (diagnostic tests AIDA VASCULITIS SCREEN, sera dilution 1:101, positive titer $>18 \mathrm{U} / \mathrm{mL}$ )

ANCA specification: sera positive in screening were further tested for antibodies against MPO and PR3 antigens using ELISA diagnostic set AIDA MPO and AIDA PR3 Sensitive. Sera dilution was 1:101 and titers > $18 \mathrm{U} /$ $\mathrm{mL}$ were considered positive in both cases

ACLA screen: anticardiolipin antibodies were tested using ELISA CardiolipinCheck tests, sera diulution 1:101, cut-off $>24.0 \mathrm{U} / \mathrm{mL}$

ACLA specification: sera positive in screening were tested for isotypes ACLA IgG and ACLA IgM with ELISA Cardiolipin GM tests, sera dilution 1:101, cut-off $>18.0 \mathrm{U} / \mathrm{mL}$

\section{Study of thyroid autoimmunity}

This part of the study, included 64 PCOS patients, age $31 \pm 4.5$ (range 23-42) years and 68 controls, average age $29 \pm 3.9$ (range 22-45) years. Blood samples were taken for the assessment of serum fT3, fT4, TSH and thyroid autoimmunity (anti-TG, anti-TPO). We also studied the prevalence of autoimmune thyroid disease (AIT). In both groups, the Diagnosis of AIT was based on elevated serum antibodies against thyroglobulin (anti-TG) and/or antibodies against thyroperoxidase (anti-TPO) with simultaneous finding of hypoechoic thyroid gland on ultrasound. FT3 and FT4 were measured by radioimmunoassay using the automatic analyser Stratec (normal ranges fT3 2.5-5.8 $\mathrm{pmol} / \mathrm{L}$, fT4 11.0-25.0 pmol/L), ultrasensitive TSH was evaluated using the kits Immunotech (France), normal range 0.17-0.45 mIU/L. Chemiluminiscent immunoanalysis was used to evaluate antithyroid autoantibodies.

Thyroid ultrasonography was performed using 2-dimensional ultrasonography ESAOTE TECHNOS MPx with linear $12 \mathrm{MHz}$ probe.

\section{Statistical analysis}

Values are given as means + SD. Groups were compared using t-tests or Mann Whitney $\mathrm{U}$ test depending on the distribution. Two-tailed Fisher's exact tests or Chisquare tests were used as appropriate for comparison of categorical variables between groups. $P$ values of $<0.05$ were considered statistically significant. For statistical analysis, we used SPSS statistical software system (SPSS 17.0; SPSS Inc., Chicago, IL). Confidence intervals were estimated using the Wilson method with continuity corrections for low expected counts. 


\section{RESULTS}

As expected, PCOS women had significantly higher BMI compared to controls $(28.08 \pm 6.91$ v.s $21.31 \pm 3.05$, $P<0.001)$, significantly higher fasting glucose level (4.81 \pm 0.98 v.s. $4.58 \pm 0.41 \mathrm{mmol} / \mathrm{L}, P=0.023)$, higher free testosterone level $(3.82+1.31$ v.s. $3.18 \pm 0.88 \mathrm{pg} / \mathrm{mL}$, $P=0.002)$ and higher $\mathrm{LH}(8.42 \pm 6.41$ v.s. $5.47 \pm 2.61$ IU/L, $P<0.001)$. We also confirmed lower levels of SHBG in PCOS women in comparison to the healthy population $(43.44 \pm 31.84$ v.s. $69.29 \pm 28 \mathrm{nmol} / \mathrm{L}, P<0.001)$.

\section{PCOS and non organ specific autoimmunity}

The prevalence of non organ specific autoantibodies in PCOS women and controls is shown in Table 1.

There were no significant differences in the prevalence of ANA screening, SSA/Ro, SSB/La, anti RNP, anti ds-DNA between PCOS and control women, which was very low in both groups. The prevalence of ANCA screen positivity was significantly higher in controls than PCOS patients $(P=0.042)$, however when specified, the frequencies of ANCA/MPO and ANCA/PR3 were similar in both evaluated groups.

There were significant differences in the positivity of ACLA IgG between examined groups, i.e. the control group had significantly higher prevalence of ACLA IgG than the PCOS women $(P=0.01)$. There were no significant differences in occurrence of the ACLA IgM positivity.

\section{PCOS and antithyroid autoimunity}

Mean values of serum TSH, FT4, anti-TG and antiTPO are demonstrated in Table 2.

There were no significant differences in serum TSH and FT4 levels between PCOS and controls. In addition serum levels of anti-TG and anti-TPO were similar in both evaluated groups.

The prevalence of anti-TG, anti-TPO positivity and the prevalence of AIT, hypo- and hyperthyroidism are shown in Table 3.

The prevalence of autoimmune thyroid disease (based on the above criteria) was slightly but not significantly

Table 1. Prevalence of non organ specific autoantibodies in PCOS women and controls.

\begin{tabular}{|c|c|c|c|c|c|}
\hline \multirow[b]{2}{*}{ Autoantibodies } & \multicolumn{2}{|c|}{$\operatorname{PCOS}(n=152)$} & \multicolumn{2}{|c|}{ Controls $(n=74)$} & \multirow[b]{2}{*}{ Significance } \\
\hline & $\begin{array}{l}\text { Number } \\
\text { of cases }\end{array}$ & $\begin{array}{l}\text { Percentage } \\
\text { prevalence } \\
(95 \% \mathrm{CI})\end{array}$ & $\begin{array}{l}\text { Number } \\
\text { of cases }\end{array}$ & $\begin{array}{c}\text { Percentage } \\
\text { prevalence } \\
(95 \% \mathrm{CI})\end{array}$ & \\
\hline ANA screen & 1 & $0.66 \%(0.03-0.417)$ & 2 & $2.70 \%(0.47-1.03)$ & $P=0.250$ \\
\hline Anti-SSA & 1 & $0.66 \%(0.03-0.417)$ & 1 & $1.35 \%(0.07-8.32)$ & $P=0.549$ \\
\hline Anti-SSB & 0 & $0 \%(0-3.07)$ & 1 & $1.35 \%(0.07-8.32)$ & $P=0.327$ \\
\hline Anti-RNP & 0 & $0 \%(0-3.07)$ & 0 & $0 \%(0-6.15)$ & NS \\
\hline Anti-dsDNA & 3 & $1.97 \%(0.51-6.11)$ & 0 & $0 \%(0-6.15)$ & $P=0.553$ \\
\hline ANCA screen & 6 & $3.95 \%(1.62-8.78)$ & 9 & $12.16 \%(6.05-22.32)$ & $P=0.042$ \\
\hline ANCA/MPO & 0 & $0 \%(0-3.07)$ & 0 & $0 \%(0-6.15)$ & NS \\
\hline ANCA/PR3 & 0 & $0 \%(0-3.07)$ & 1 & $1.35 \%(0.07-8.32)$ & $P=0.327$ \\
\hline ACLA IgG & 0 & $0 \%(0-3.07)$ & 4 & $5.40 \%(1.75-13.99)$ & $P=0.011$ \\
\hline ACLA IgM & 1 & $0.66 \%(0.03-0.417)$ & 3 & $4.05 \%(1.05-12.18)$ & $P=0.104$ \\
\hline
\end{tabular}

Table 2. Mean serum levels of thyroid parameters in PCOS women and controls.

\begin{tabular}{lccl}
\hline Mean serum levels & PCOS $(\mathrm{n}=64)$ & Controls $(\mathrm{n}=68)$ & Significance \\
\hline TSH $(\mathrm{mIU} / \mathrm{L})$ & $2.37 \pm 1.46$ & $2.37 \pm 1.46$ & $P=0.937$ \\
TT4 $(\mathrm{pmol} / \mathrm{L})$ & $16.36 \pm 5.34$ & $16.49 \pm 2.32$ & $P=0.852$ \\
aTG $(\mathrm{IU} / \mathrm{mL})$ & $53.09 \pm 157.07$ & $29.8 \pm 100.77$ & $P=0.386$ \\
aTPO $(\mathrm{IU} / \mathrm{mL})$ & $59.74 \pm 149.03$ & $45 \pm 204.77$ & $P=0.805$ \\
\hline
\end{tabular}

Table 3. Prevalence of antithyroid antibodies, AIT and thyroid dysfunction in PCOS women and controls.

\begin{tabular}{lccccc}
\hline \multirow{2}{*}{ Prevalence } & \multicolumn{2}{c}{ PCOS $(\mathrm{n}=64)$} & \multicolumn{2}{c}{ Controls $(\mathrm{n}=68)$} & Significance \\
\cline { 2 - 5 } & $\begin{array}{l}\text { Number } \\
\text { of cases }\end{array}$ & $\begin{array}{l}\text { Percentage } \\
\text { prevalence }\end{array}$ & $\begin{array}{l}\text { Number } \\
\text { of cases }\end{array}$ & $\begin{array}{l}\text { Percentage } \\
\text { prevalence }\end{array}$ & \\
\hline + aTG & 5 & $7.81 \%$ & 3 & $4.41 \%$ & $P=0.293$ \\
+ aTPO & 12 & $18.75 \%$ & 5 & $7.35 \%$ & $P=0.045$ \\
AIT & 12 & $18.75 \%$ & 7 & $10.29 \%$ & $P=0.163$ \\
Hypothyroidism & 7 & $10.94 \%$ & 9 & $13.24 \%$ & $P=0.882$ \\
Hyperthyroidism & 1 & $1.56 \%$ & 0 & $0 \%$ & $P=0.301$ \\
\hline
\end{tabular}


higher in PCOS patients. Moreover, there were no significant differences in the occurrence of thyroid dysfunction ( $P=0.082$ for hypothyroidism, and $P=0.301$ for hyperthyroidism) between groups. The frequency of anti-TG positivity was similar in PCOS and controls however the prevalence of anti-TPO positivity was significantly higher in PCOS women. The relative risk for positivity of aTG and aTPO in patients with PCOS in comparison with healthy women was better estimated by calculating the odds ratios with 95\% CI using the Mantel-Haenszel test. As shown in Table 4, women with PCOS had a more than 2fold higher risk for AIT and almost a double risk for positivity of both types of anti thyroid antibodies.

\section{DISCUSSION}

According to the best our knowledge this is the biggest study on autoimmunity in PCOS patients and the widest spectrum of non organ specific autoantibodies examined to date. The results did not confirm any higher frequency of non organ specific autoantibodies in PCOS women than healthy women. Moreover, there was significantly higher prevalence of ACLA IgG antibodies in controls, suggesting that the hyperandrogenic state in PCOS may be a protective factor against the autoimmune response.

Data on the prevalence of non organ-specific autoimmune disorders in PCOS are poor and controversial. There is evidence that estrogen excess has been linked to different autoimmune diseases ${ }^{11-13}$. Because of chronic anovulation and luteal insufficiency, PCOS women have relative hyperestrogenism which could promote the autoimmune response. On the other hand, high levels of androgens presenting in these patients seem to have a protective role in the development of autoimmune diseases, which is in agreement with our results.

There are few papers demonstrating the presence of various non organ-specific antibodies in PCOS women. Former studies on relatively small groups of patients demonstrated a higher prevalence of ANA positivity in PCOS women $^{14,15}$. Recently, Hefler-Frishmuth et al., evaluated sera from PCOS patients for the presence of non organspecific autoantibodies (ANA, anti-histone, anti-nucleosome and anti-dsDNA). Statistical significance was found only in the levels of anti-histone and anti dsDNA antibodies $^{16}$. In another study, a significantly higher prevalence of ANA and SMA was found in PCOS patients compared to controls ( $19.4 \%$ v.s. $5.1 \%)$. The prevalence of other antibodies was very low and similar to that in the control group ${ }^{14}$. This is particularly in contrast to our study, demonstrating very low occurrence of the ANA positivity in the PCOS group. The prevalence of anti SSA, SSB, anti dsDNA, anti RNP antibodies was also very low and comparable with the results of above mentioned study.

Although ANCA screen in our study demonstrated higher frequency of positivity in controls, further evaluating of ANCA (ANCA/MPO) did not detect any differences between PCOS and controls. In contrast we found a higher prevalence of ACLA IgG in control group as
Table 4. The relative risk for positivity of aTG and aTPO in patients with PCOS in comparison with healthy women.

\begin{tabular}{lcl}
\hline & OR & $95 \% \mathrm{CI}$ \\
\hline aTG & 1.836 & $0.42-8.019$ \\
aTPO & 2.988 & $0.986-9.056$ \\
Hypothyroidism & 0.922 & $0.314-2.710$ \\
\hline Hyperthyroidism & NA & \\
\hline AIT & 2.244 & $0.829-6.071$ \\
\hline
\end{tabular}

compared to PCOS women. Recently one study showed lower levels of IgM anti-phosphorylcholine antibodies in PCOS patients as compared to healthy women which is in agreement with our results ${ }^{16}$. All these findings might indicate that PCOS women could be protected against autoimmune diseases, which is probably related to hyperandrogenemia.

In the second part of this study we demonstrated similar plasma levels of fT4, TSH and also significantly higher prevalence of anti-TPO positivity in patients compared to controls. The overall prevalence of AIT was slightly but not significantly higher in PCOS women. Previous data on the association between PCOS and AIT are controversial. Some reports documented higher prevalence of AIT and higher levels of TSH in PCOS women. Although we found $13 \%$ prevalence of hypothyroidism in apparently healthy women, some data suggest that up to $18 \%$ of the general population might suffer from hypothyroidism ${ }^{17}$. In a Chinese population of PCOS women higher levels of TSH were documented only in women with a $\mathrm{LH} / \mathrm{FSH}$ ratio over 3 (ref. ${ }^{18}$ ). Janssen et al. presented significantly higher prevalence of anti-TPO and/or anti-TG antibodies in PCOS patients, higher prevalence of AIT and also higher levels of TSH in PCOS women ${ }^{19}$. Comparably, significantly higher titers of anti-TPO antibodies and a higher prevalence of goiter with AIT characteristics were shown in 78 PCOS Iranian women compared to a healthy population $^{20}$. In contrast, a higher prevalence of PCOS in euthyroid girls with AIT has been demonstrated in a recent study ${ }^{21}$. These data suggest a possible role of autoimmunity in the etiopathogenesis of PCOS but this hypothesis is unproven as a recent study clearly documented that PCOS alone was not associated with thyroid disease and the metabolic syndrome or its components appear to be related to thyroid volume, function and antithyroid antibody levels ${ }^{22}$. Because the BMI in our patients was significantly higher than in controls, we assume that slight differences in the occurrence of anti-TPO prevalence may be related to obesity and insulin resistance.

Finally an autoimmune mechanism of PCOS has been suggested by some authors. According to Gleicher et al. this disease might be an opposite condition to premature ovarian failure ${ }^{23}$. Suh et al. reported histological findings in a case of PCOS consistent with autoimmune oophoritis $^{24}$ and Lee et al. described a case of a patient with combined PCOS and autoimmune polyglandular syndrome type 2 (ref. ${ }^{25}$ ). Recently Chapman described a fundamental role of thymus and $\mathrm{T}$ cells in estrogen 
induced follicle cyst formation ${ }^{26}$. However studies of anti-ovarian antibodies performed so far have yielded conflicting results. The group of Lonsdale demonstrated ovarian antibodies, and lymphocytic infiltration of ovaries in two patients with PCOS (ref. ${ }^{27}$ ). Some others showed antibodies to human ovarian sections and granulosa cells in patients with PCOS (ref. ${ }^{28,29}$ ). Many other targeting antigens of ovarian immune pathology have been described in PCOS women ${ }^{30}$. In another study, positive anti-ovarian antibodies for at least one isotype were present in $44 \%$ of the PCOS women ${ }^{31}$. However these results could not be confirmed by others. Nevertheless this aspect of PCOS pathogenesis is still open for further research and requires further studies of other antibodies against various ovarian structures.

We conclude that the prevalence of non organ-specific autoantibodies in PCOS women is very low and similar or even lower than in healthy controls. On the other hand, we found a slightly higher prevalence of thyroid autoimmuinity in PCOS women but this finding may be related to the metabolic syndrome rather than to PCOS alone.

\section{ACKNOWLEDGEMENT}

Author contributions: JP, JF: manuscript drafting; JV: supplied sera from PCOS pacients; ID: provided PCOS pacients; DK, ZV, JR: laboratory investigations; IL: manuscript revising.

Conflict of interest statement: None declared.

\section{REFERENCES}

1. Fauser BC, Tarlatzis BC, Rebar RW, Legro RS, Balen AH, Lobo R Carmina E, Chang J, Yildiz BO, Laven JS, Boivin J, Petraglia F, Wijeyeratne CN, Norman RJ, Dunaif A, Franks S, Wild RA, Dumesic $\mathrm{D}$, Barnhart K. Consensus on women's health aspects of polycystic ovary syndrome (PCOS): the Amsterdam ESHRE/ASRM-Sponsored 3rd PCOS Consensus Workshop Group. Fertil Steril 2012;97:28-38.

2. Azziz R, Carmina E, Dewailly D, Diamanti-Kandarakis E, EscobarMorreale HF, Futterweit W, Janssen OE, Legro RS, Norman RJ, Taylor $A E$, Witchel SF, Androgen Excess Society. Positions statement: criteria for defining polycystic ovary syndrome as a predominantly hyperandrogenic syndrome: an Androgen Excess Society Guideline. J Clin Endocrinol Metab 2006;91:4237-45.

3. Ehrmann DA, Barnes RB, Rosenfeld RL, Cavaghan MK, Imperial J. Prevalence of impaired glucose tolerance and diabetes in women with polycystic ovary syndrome. Diabetes Care 1999;22:141-6.

4. De Ugarte CM, Bartolucci AA, Azziz R. Prevalence of insulin resistance in the polycystic ovary syndrome using the homeostasis model assessment. Fertil Steril 2005;83:1454-60.

5. van Gelderen CJ, Gomes dos Santos M. Polycystic ovarian syndrome. Evidence for an autoimmune mechanism in some cases. J Reprod Med 1993;38:381-6.

6. Rojanski N, Roll D, Meirow D. Polycystic ovary syndrome. An autoimmune disease? J Reprod Med 1997;42:325.

7. Shoenfeld Y, Zandman-Goddard G, Stojanovich L, Cutolo M, Amital $\mathrm{H}$, Levy Y, Abu-Shakra M, Barzilai O, Berkun Y, Blank M, de Carvalho JF, Doria A, Gilburd B, Katz U, Krause I, Langevitz P, Orbach H, Pordeus V, Ram M, Toubi E, Sherer Y. The mosaic of autoimmunity: hormonal and environmental factors involved in autoimmune diseases-2008. Isr Med Assoc J 2008;10:1:8-12.

8. Angstwurm MW, Gartner R, Ziegler-Heitbrock HW. Cyclic plasma IL-6 levels during normal menstrual cycle. Cytokine 1997;9:370-4.
9. Petríková J, Lazúrová I, Shoenfeld Y. Polycystic ovary syndrome and autoimmunity. Eur J Intern Med 2010; 21:367-1.

10. Niccoli G, Apa R, Lanzone A, Liuzzo G, Spaziani C, Sagnella F, Cosentino N, Moro F, Martinez D, Morciano A, Bacà M, Pazzano V, Gangale MF, Tropea A, Crea F. CD4+CD28 null T lymphocytes are expanded in young women with polycystic ovary syndrome. Fertil Steril 2011;95(8):2651-4.

11. Lahita RG. Gender disparity in systemic lupus erythematodes, thoughts after the 8th International Congress on SLE. Shanghai, China, 2007. J Clin Rheumatol 2008;14:185-7.

12. Li J, Su JM, Zeng XJ. Is primary biliary cirrhosis another example of an immunemediated complication of Klinefelter syndrome? J Clin Rheumatol 2004;10:286-7.

13. Rovenský J, Imrich R, Lazúrová I, Payer J. Rheumatic Diseases and Klinefelter's Syndrome. Ann N Y Acad Sci 2010;1193:1-9.

14. Reimand K, Talja I, Metsküla K, Kadastik U, Matt K, Uibo R. Autoantibody studies of female patients with reproductive failure. J Reprod Immunol 2001;51:167-76.

15. Hefler-Frischmuth K, Walch K, Huebl W, Baumuehlner K, Tempfer $C$, Hefler L. Serologic markers of autoimmunity in women with the polycystic ovary syndrome. Fertil Steril 2009;92(5):60.

16. Gingnell M, Dahlbom I, Lindholm A, Hudecova M, Arnadottir R, Hansson T, Sundstrom-Poromaa I. Patients with polycystic ovary syndrome have lower levels of IgM anti-phosphorylcholine antibodies than healthy women. Gynecol Endocrinol 2011;27(7):486-90.

17. American Thyroid Association Website. "ATA Hypothyroidism Booklet." Falls Church, VA: American Thyroid Association. 2003. Retrieved Nov. 14, 2006, from http://www.thyroid.org/patients/ brochures/Hypothyroidism\%20_web_booklet.pdf.

18. Wu X, Zhang Z, Su Y. Functional states of pituitary-ovary, -adrenal and -thyroid axes in women with polycystic ovary syndrome. Zhonghua Fu Chan Ke Za Zhi 1998;33:153-6.

19. Janssen OE, Mehlmauer N, Hahn S, Offner AH, Gärtner R. High prevalence of autoimmune thyroiditis in patients with polycystic ovary syndrome. Eur J Endocrinol 2004;150:363-9.

20. Kachuei M, Jafari F, Kachuei A, Keshteli AH. Prevalence of autoimmune thyroiditis in patients with polycystic ovary syndrome. Arch Gynecol Obstet 2012;285:853-6.

21. Ganie MA, Marwaha RK, Aggarwal R, Singh S. High prevalence of polycystic ovary syndrome characteristics in girls with euthyroid chronic lymphocytic thyroiditis: a case-control study. Eur J Endocrinol 2010;162(6):1117-22.

22. Anaforoglu I, Topbas M, Algun E. Relative association of polycystic ovarian syndrome v.s. metabolic syndrome with thyroid function, volume, nodularity and autoimmunity. J Endocrinol Invest 2011;34:259-64.

23. Gleicher N, Barad DH. Gender as risk factor for autoimmune diseases. J Autoimmun 2007;28:1-6.

24. Suh Y. Autoimmune oophoritis-a case report. J Korean Med Sci $1992 ; 7: 284-90$

25. Lee SH, Kim MR, Kim JH, Kwon HS, Yoon KH, Son HY, Cha BY. A patient with combined polycystic ovary syndrome and autoimmune polyglandular syndrome type 2. Gynecol Endocrinol 2007;23:252-6.

26. Chapman JC, Min SH, Freeh SM, Michael SD. The estrogen-injected female mouse: new insight into the etiology of PCOS. Reprod Biol Endocrinol 2009;18;7:47.

27. Lonsdale RN, Roberts PF, Trowell JE. Autoimmune oophoritis associated with polycystic ovaries. Histopathol 1991;19:77-81.

28. van Gelderen CJ, Gomes dos Santos M. Polycystic ovarian syndrome. Evidence for an autoimmune mechanism in some cases. J Reprod Med 1993;38:381-6.

29. Luborsky JL, Shatavi S, Adamczyk P, Chiong C, Llanes B, Lafniztzegger J, Soltes B, McGovern P, Santoro N. Polycystic ovary syndrome and ovarian autoimmunity- assessment of ovarian antibodies by EIA. J Reprod Immunol 1999;42:79-84.

30. Forges T, Monnier-Barbarino P, Faure CG, Béné MC. Autoimmunity and antigenic targets in ovarian pathology. Hum Reprod Update 2004; 10:163-75.

31. Fénichel $P$, Gobert $B$, Carré $Y$, Barbarino-Monnier $P$, Hiéronimus S. Polycystic ovary syndrome in autoimmune disease. Lancet 1999;353:2210. 